INPLASY

PROTOCOL

To cite: Deo et al. The timevarying cardiovascular benefits of glucagon like peptide-1 agonist (GLP-RA)therapy in patients with type 2 diabetes mellitus: A meta-analysis of multinational randomized trials. Inplasy protocol 202170097. doi:

10.37766/inplasy2021.7.0097

Received: 30 July 2021

Published: 30 July 2021

Corresponding author:

Salil Deo

s.deo.1@research.gla.ac.uk

Author Affiliation:

University of Glasgow.

Support: None.

Review Stage at time of this submission: Preliminary searches.

Conflicts of interest:

None declared.

\section{The time-varying cardiovascular benefits of glucagon like peptide-1 agonist (GLP-RA)therapy in patients with type 2 diabetes mellitus: A meta-analysis of multinational randomized trials}

Deo, SV1; McAllister, DA2; Sattar, N3; Pell, JP4.

Review question / Objective: P - patients with type 2 diabetes mellitus already receiving routine medical therapy; I - patients receiving glucagon like peptide 1 receptor agonist (GLP1 receptor agonist) therapy (semaglutide, dulaglutide, liraglutide, exenatide, lixisenatide, efpeglenatide, abiglutide); C - patients receiving standard therapy for diabetes mellitus but not receiving GLP1 agonist therapy; 0 - composite end point as per invididual trial, cardiovascular mortality, all-cause mortality, myocardial infarction, stoke.

Condition being studied: Type 2 diabetes mellitus.

Study designs to be included: Randomised controlled trials which enroll a large number of patients (defined as $>500$ ) and are multinational in origin. Studies included will need to have published Kaplan and Meier curves for the end-points presented in the manuscript.

INPLASY registration number: This protocol was registered with the International Platform of Registered Systematic Review and Meta-Analysis Protocols (INPLASY) on 30 July 2021 and was last updated on 30 July 2021 (registration number INPLASY202170097).

\section{INTRODUCTION}

Review question / Objective: P - patients with type 2 diabetes mellitus already receiving routine medical therapy; I patients receiving glucagon like peptide 1 receptor agonist (GLP1 receptor agonist) therapy (semaglutide, dulaglutide, liraglutide, exenatide, lixisenatide, efpeglenatide, abiglutide); C - patients receiving standard therapy for diabetes mellitus but not receiving GLP1 agonist therapy; $O$ - composite end point as per invididual trial, cardiovascular mortality, all- 
cause mortality, myocardial infarction, stoke.

Rationale: The study will apply the restricted mean survival time (RMST) method to randomised trials. Using published Kaplan Meier curves for each trial and for each end-point, data will be abstracted. Difference in RMST will be obtained and pooled using a random effects model. The study will provide an easy to understand estimate of the possible benefit of using GLP1 RA on cardiovascular outcomes.

Condition being studied: Type 2 diabetes mellitus.

\section{METHODS}

Search strategy: ("glucagon-like peptide-1 receptor agonist", OR "albiglutide",OR "dulaglutide",OR "efpeglenatide",OR

"exenatide", OR "liraglutide",OR "lixisenatide",OR "semaglutide")AND ( "randomized clinical trial") AND ("death",OR "myocardial infarction",OR "stroke",OR "heart failure" OR "kidney").

Participant or population: Patients being treated for type 2 diabetes mellitus.

Intervention: Patients receiving glucagon like peptide 1 receptor agonist (GLP1 agonist) therapy (semaglutide, dulaglutide, liraglutide, exenatide, lixisenatide, efpeglenatide, abiglutide).

Comparator: Patients receiving routine care for type 2 diabetes mellitus and any other medical conditions that they may have.

Study designs to be included: Randomised controlled trials which enroll a large number of patients (defined as $>500$ ) and are multinational in origin. Studies included will need to have published Kaplan and Meier curves for the end-points presented in the manuscript.

Eligibility criteria: (1) Randomised control trials using GLP1 agonist agents to present clinical results of adult human subjects. (2)
Trials should be multi-national and contain $>500$ participants (3) Trials that contain a cardiovascular end-point (major adverse cardiovascular events, stroke, myocardial infarction, cardiovascular mortality, allcause mortality) specified above will be included.

Information sources: Medline (Pubmed) from inception till July 30th 2021.

Main outcome(s): composite cardiovascular adverse events as per trial; all cause mortality; cardiovascular mortality; myocardial infarction; stroke.

\section{Additional outcome(s): None.}

Data management: Data will be stored in a central repository accessed by all authors. Data will be abstracted using a prespecified form. All Kaplan and Meier plots will be selected from included trials. Disagreement with data collection results will be resolved by discussion with senior authors (NS,JP).

Quality assessment / Risk of bias analysis: Heterogeniety will be evaluated for each end-point using 12 method. Cochran risk of bias tool will be utilised to evaluate risk of bias for included studies.

Strategy of data synthesis: Kaplan Meier plots from included trials will be collected. Data will be extracted using Scan IT software and the Guyot method. From the extracted data, a flexible parametric model will be fit for each trial curve. The RMST will be calculated for each trial included in that particular end-point. Data will be pooled using the random effects models and the DerSimonian and Laird method. Heterogeneity will be evaluated by calculating the 12. Results will be presented at $12,24,36$ and 48 months for the endpoint of MACE. For mortality, CV mortality and myocardial infarction, results will be presented at 24 and 48 months.

Subgroup analysis: None planned.

Sensitivity analysis: Sensitivity analysis will be performed by using univariate direct 
method rather than the parametric method. Effect estimates obtained from both models will be compare graphically to evaluate for overlap.

Language: English.

Country(ies) involved: United States.

Other relevant information: None.

Keywords: diabetes mellitus; glucagon like peptide 1 receptor agonist; cardiovascular outcomes.

Dissemination plans: Study will be published in a peer reviewed journal and also submitted as an abstract for conference presentation.

Contributions of each author:

Author 1 - Salil Deo.

Email: s.deo.1@research.gla.ac.uk

Author 2 - David McAllister - study design, review of statistical analyses and critical appraisal of manuscript - review of manuscript.

Email: david.mcallister@glasgow.ac.uk

Author 3 - Naveed Sattar - study conception, design, interpretation of results, review and critical appraisal of manuscript.

Email: naveed.sattar@glasgow.ac.uk

Author 4 - Jill Pell - study conception, study design, review of manuscript, critical appraisal of manuscript, interpretation of results.

Email: jill.pell@glasgow.ac.uk 